\title{
INSTRUMENTO DE CLASSIFICAÇÃO DE PACIENTES DE PERROCA : TESTE DE CONFIABILIDADE PELA CONCORDÂNCIA ENTRE AVALIADORES - CORRELAÇÃO ${ }^{1}$
}

\author{
INTERRATER RELIABILITY OF THE PERROCA 'S INSTRUMENT FOR \\ PATIENTS CLASSIFICATION: ASSESSMENT
}

\section{INSTRUMENTO DE PERROCA PARA LA CLASIFICACIÓN DE PACIENTES: TEST DE FIABILIDAD POR LA CONCORDANCIA ENTRE LOS EVALUADORES CORRELACIÓN}

Marcia Galan Perroca* Raquel Rapone Gaidzinski**

Perroca MG, Gaidzinski. Instrumento de classificação de pacientes de Perroca: teste de confiabilidade pela concordância entre avaliadores - correlação. Rev Esc Enferm USP 2002; 36(3): 245-52.

\begin{abstract}
RESUMO
Esta pesquisa foi conduzida com o intuito de estudar, analisar e testar a confiabilidade do instrumento de classificação de pacientes proposto por Perroca (', ,baseado nas necessidades individualizadas de cuidado de enfermagem. O instrumento foi aplicado pelas enfermeiras lotadas na UTI de um hospital de ensino, em 50 pacientes escolhidos aleatoriamente. O coeficiente de correlação de Pearson foi utilizado para a avaliação do grau de concordância nos escores totais obtidos pelas enfermeiras. A análise de confiabilidade revelou que as correlações são altas e positivas entre os escores dados pelas enfermeiras.
\end{abstract}

PALAVRAS-CHAVE: Avaliação em enfermagem. Confiabilidade e validade.

\begin{abstract}
The purpose of this research was to check the reliability of Perroca's instrument model for classifying patients, based on the particular needs of nursing care. This patients' classification instrument was applied in 50 patients randomly chosen by the nurses of the General Intensive Care Unit of a medical school hospital. Pearson's quotient correlation was used to analyze the concordance degree of the total scores by the nurses. The reliability analysis showed a high and positive correlation in relation to scores given by the nurses.
\end{abstract}

KEYWORDS: Nursing assessment. Reproducibility of results.

\section{RESUMEND}

Esta investigación fue conducida con la intención de estudiar, analizar y probar la fiabilidad Del instrumento propuesto por Perroca(1) para la clasificación del pacientes basado en las necesidades individualizadas de cuidados de enfermaria. El instrumento fue aplicado por las enfermeras asignadas en la UCI de un hospital de instrucción, en 50 pacientes escogidos de forma aleatoria. Para verificar el grado de acuerdo entre las puntuaciones dadas por las enfermeras se usó el coeficiente de correlación de Pearson. El análisis de fiabilidad reveló que las correlaciones entre las puntuaciones dadas por las enfermeras son altas y positivas.

PALABRAS -CLAVE: Evaluación en enfermería. Reproducibilidad de resultados.

\footnotetext{
$1 \quad$ Parte da Tese de Doutorado apresentada à EEUSP-SP, 2001.

* Doutora em Enfermagem. Docente do Departamento de Enfermagem Especializada da FAMERP, S.J. Rio Preto; E-mail: marcgap@zaz.com.br.

** Professor Livre Docente do Departamento de Orientação Profissional da EEUSP-SP. Orientadora
} 


\section{INTRODUÇÃO}

No desempenho de sua prática profissional, compete ao enfermeiro o gerenciamento da assistência de enfermagem prestada ao paciente. Contudo, no contexto hospitalar, o enfermeiro ainda tem desenvolvido um gerenciamento mais voltado para as necessidades de serviço e para o cumprimento de regulamentos, normas e tarefas atuando como mero executor do que é preconizado pela organização e pelos profissionais da equipe médica. Este estilo de gerenciamento não contribui para uma atuação direcionada ao conhecimento e atendimento das reais necessidades cuidativas dos pacientes( ${ }^{(2)}$.

Em sua prática administrativa, os enfermeiros necessitam lançar mão de instrumentos que os auxiliem a gerenciar o cuidado prestado ao paciente.

Nesse sentido, o Sistema de Classificação de Pacientes (SCP) constitui-se em um instrumento valioso, na medida em que disponibiliza dados das condições do paciente, os quais vão auxiliar no processo decisório relacionado à alocação de recursos humanos, qualidade da assistência, monitorização da produtividade e processo orçamentário .(3-5)

O SCP é um processo no qual se procura categorizar pacientes de acordo com a quantidade de cuidado de enfermagem requerida, ou seja, baseada no grau de complexidade da assistência de enfermagem.

Com o objetivo de nortear a classificação de pacientes por tipo de cuidado, Perroca (1) e Perroca; Gaidzinski (8) construíram um instrumento baseado nas necessidades individualizadas de cuidado de enfermagem. Na elaboração deste instrumento optouse por uma estruturação mediada pela avaliação de indicadores, baseados nas necessidades humanas básicas preconizadas por Horta (7)

O instrumento utiliza 13 indicadores críticos que não se restringem apenas à esfera biológica, mas que consideram também a dimensão psicossocial do cuidado. Os 13 indicadores críticos considerados para compor o instrumento são: Estado Mental e Nivel de Consciência; Oxigenação; Sinais Vitais; Nutrição e Hidratação; Motilidade; Locomoção; Cuidado Corporal; Eliminações; Terapêutica; Educação à Saúde; Comportamento; Comunicação e Integridade Cutâneo- Mucosa.

A terminologia indicadores criticos foi adotada para designar as necessidades de cuidado de enfermagem do paciente que, quando associadas entre si, fossem capazes de identificar a categoria de cuidados a que este paciente pertence.
Cada um dos indicadores possui gradação de 1 a 5 , objetivando apontar a intensidade crescente de complexidade do cuidado, de forma que, o valor 1 corresponde ao menor nivel de atenção de enfermagem e o valor 5, ao nível máximo de complexidade assistencial.

O paciente é classificado em todos os indicadores, em um dos cinco níveis, na opção que melhor descreva a sua situação com relação à assistência de enfermagem.

O valor obtido individualmente, em cada um dos indicadores, é então somado e o total obtido é comparado com pontuações existentes em um quadro conduzindo, dessa forma, a uma classe ou categoria de cuidado a que este paciente pertence, como segue: cuidados mínimos (13 a 26 pontos), cuidados intermediários (27 a 39 pontos), cuidados semiintensivos (40 a 52 pontos) e cuidados intensivos (53 a 65 pontos).

O instrumento de classificação de pacientes de Perroca (1) apresenta os seguintes intervalos de pontuações nas quatro categorias de cuidado: cuidados mínimos $13 a 26$ pontos; cuidados intermediários 27 a 39 pontos; cuidados semi-intensivos 40 a 52 pontos $e$ cuidados intensivos 53 a 65 pontos.

Para Giovannetti (8), Giovannetti; Mayer(9),

Whitney; Killien(10), Haas (11), Williams (12), De

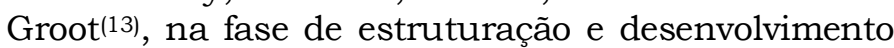
de um instrumento para classificação de pacientes, torna-se questão essencial estabelecer e monitorar a sua confiabilidade e validade.

Para mensurar a validade do instrumento de classificação proposto, ou seja, para se verificar a confiabilidade do instrumento em medir o que se propõe a medir, utilizou-se, em primeira instância, o método de validação de conteúdo através da aplicação da Técnica Delphi (14), com o objetivo de avaliar a extensão da dependência do paciente com relação à assistência de enfermagem.

Após a validação do conteúdo do instrumento de classificação de pacientes pelos juízes, emergiu a necessidade de se analisar a confiabilidade e a validade de conteúdo do instrumento, verificando se ele apresenta exatidão no seu uso. Esta monitorização possibilitará a aceitação do instrumento pelos enfermeiros de serviço e favorecerá a credibilidade das informações fornecidas pelo mesmo.

Dessa forma, este estudo se propõe a :

- Realizar teste de confiabilidade do instrumento através do coeficiente de correlação de Pearson 


\section{SISTEMA DE CLASSIFICAÇÃO DE PACIENTES: IMPORTÂNCIA NA PRATICA GERENCIAL}

Os gerentes de enfermagem podem utilizar $o$ SCP como instrumento para :

- Proporcionar meio objetivo e científico para identificação das necessidades de atenção de enfermagem aos pacientes;

- Nortear a alocação quanti/qualitativa de recursos humanos para a assistência de enfermagem;

- Fortalecer a argumentação no processo de negociação com a administração;

- Favorecer a obtenção de dados referentes a qualidade do cuidado proporcionado;

- Obter eficaz sistema de comunicação das diferentes necessidades de atenção de enfermagem dos pacientes,

- Nortear a organização dos serviços, o processo de tomada de decisão e o planejamento da assistência de enfermagem;

- Caracterizar a clientela usuária dos serviços estabelecendo o perfil do cliente para cada tipo de cuidado ou grau de dependência;

- Auxiliar no processo orçamentário do Servico de Enfermagem apontando a diferente intensidade de utilização dos recursos na assistência.

\section{TRAJETÓRIA METODOLÓGICA}

O teste de confiabilidade do instrumento de classificação de pacientes, nessa investigação, compreendeu a análise da variação dos escores totais emitidos pelas enfermeiras (em si mesmo) para se verificar a existência de discrepância entre eles (Pearson).

No que diz respeito aos aspectos éticos envolvidos na realização dessa pesquisa, cumpre esclarecer que a coleta de informações somente foi iniciada após parecer favorável do Comitê de Ética em Pesquisa da instituição campo de estudo.

Após explanação dos objetivos dessa investigação, foi solicitada aprovação formal da Gerência de Enfermagem, bem como o consentimento livre e esclarecido das enfermeiras atuantes na Unidade de Terapia Intensiva Geral para participarem da pesquisa.

\section{Testes de Confiabilidade}

Um dos principais critérios para avaliação da qualidade de um instrumento de mensuração, para
Polit; Hungler (15), é a sua confiabilidade e esta pode ser avaliada pelo grau de coerência com o qual este instrumento mede o atributo. A confiabilidade será considerada maior quando, em repetidas mensurações de um atributo, se obtiver uma menor variação. Podese também dizer, que um instrumento é confiável considerando-se o seu grau de precisão, ou seja, quando suas medidas conseguem refletir, de forma precisa, as medidas reais do atributo investigado.

A determinação da confiabilidade fornece uma indicação da extensão na qual a medida de um instrumento admite erros variáveis em seu uso. A confiabilidade pode ser avaliada tendo-se em conta 3 aspectos: a estabilidade ou consistência todo o tempo; a equivalência ou consistência entre várias versões ou aplicações e a consistência interna ou homogeneidade que se refere à consistência dentro da escala ou instrumento (15-16)

Para Whitney; Killien(10) a confiabilidade de um instrumento de classificação relaciona-se com a consistência apresentada nos resultados obtidos quando diferentes enfermeiros aplicam o instrumento na avaliação de pacientes.

De acordo com Giovannetti; Mayer(9) e Whitney; Killien(10) vários enfoques estatísticos podem ser utilizados para se determinar a confiabilidade entre avaliadores.

Entre os métodos mais usualmente empregados podem ser citados: 1- a porcentagem de concordância entre "especialistas" e enfermeiros da unidade; 2- o indice Kappa e 3- 0 Coeficiente de Correlação de Pearson. A escolha do método depende do tipo de sistema de classificação empregado e de seus dados resultantes, dos recursos e especialistas disponíveis, como também de rigor metodológico.

O procedimento de correlação constitui-se no método mais comum para se descrever a associação entre variáveis quantitativas.

Explicam Polit; Hungler(17) que os coeficientes de correlação são, normalmente, designados com um $r$ e costumam variar de $+1,00$ (para uma relação direta perfeita) passando por 0,00 (ausência de relação) e chegando a -1,00 (para uma relação inversa perfeita). Desta forma, quanto mais elevado for o valor obtido, mais confiável pode ser considerado o instrumento.

O coeficiente de correlação mais usado, designando a magnitude de relação entre duas variáveis, mensuradas em pelo menos, uma escala por intervalo é o chamado coeficiente de correlação ou $r$ de Pearson.

Os valores e escores obtidos quando da utilização de um instrumento, por melhor que ele seja considerado, apresentam determinado grau de erro. 
Cada escore é constituído por duas partes: um componente de erro (escore observado) e um componente de verdade (escore real). O escore real não pode ser conhecido, desde que as medidas não são infaliveis $(15)$.

Operacionalização do Teste de Confiabilidade pela concordância entre avaliadores

O instrumento de classificação de pacientes foi aplicado na Unidade de Terapia Intensiva Geral de um hospital de ensino - o Hospital de Base, entidade de direito privado, filantrópico, sem fins lucrativos, da cidade de São José do Rio Preto, estado de São Paulo. O hospital possui 637 leitos.

Optou-se por realizar o teste de confiabilidade na Unidade de Terapia Intensiva Geral por ser a única unidade de internação na instituição, na qual atuam 4 enfermeiras no mesmo turno de trabalho - condição esta indispensável para a efetivação do teste de confiabilidade selecionado para este estudo, qual seja, a concordância entre as avaliações das enfermeiras.

A Unidade de Terapia Intensiva Geral é formada por 24 leitos destinados a pacientes adultos distribuídos em duas unidades distintas: a UTI Geral I e a UTI Geral II.

A UTI recebe tanto pacientes que demandam nível de atenção de enfermagem intensiva como também semi-intensiva.

Os sujeitos foram as quatro enfermeiras lotadas no plantão da manhã que anuíram em participar do estudo, sendo : uma enfermeira supervisora, uma enfermeira assistencial e duas aprimorandas de enfermagem, as quais doravante serão designadas por enfermeira $V$, enfermeira $L$, enfermeira $R e$ enfermeira MS.

\section{Procedimento de Coleta de Informações}

Estas enfermeiras atuaram como avaliadoras para classificar o grau de complexidade assistencial de 50 pacientes, internados nessa unidade, escolhidos aleatoriamente, no período de 08 a 30 de junho de 1999. 0 tamanho amostral foi definido estatisticamente.

Diariamente, de segunda a sexta-feira a pesquisadora selecionava, aleatoriamente, 5 leitos das Unidades de Terapia Intensiva cujos pacientes deveriam ser classificados pelas enfermeiras. Este procedimento continuou até que se completasse um total de 50 pacientes classificados. A classificação dos pacientes algumas vezes sofreu interrupções, devido às folgas e mobilizações das enfermeiras para outras unidades.

Visto se tratar de um teste de confiabilidade, em que a interpretação dos sujeitos sobre o instrumento utilizado é parte fundamental dos resultados, as orientações foram breves e sucintas.

Nesta oportunidade, foi entregue às enfermeiras um formulário para registro dos dados. Esse formulário foi composto de duas partes: a primeira abrangeu os dados de identificação do paciente (nome, idade, registro, unidade, quarto, leito), data e horário da coleta de dados. A outra parte referiu-se ao levantamento de informações voltadas diretamente à classificação do grau de complexidade assistencial dos pacientes internados com espaço destinado à colocação do escore obtido pelas enfermeiras avaliadoras, individualmente, em cada um dos indicadores, do total da pontuação obtida, a categoria de cuidado à qual o paciente pertencia, bem como, o nome da enfermeira que procedeu a classificação.

Solicitou-se a cada enfermeira avaliadora, a aplicação do instrumento sob forma de exame fisico e entrevista aos pacientes, cujos leitos estavam assinalados no formulário, no mesmo turno de trabalho, em horários os mais próximos possivieis.

As enfermeiras foram orientadas a não consultarem as demais colegas durante este processo, assim como, para não comunicarem às demais, os escores obtidos em suas avaliações a fim de se evitar influências nas respostas.

Os formulários devidamente preenchidos eram recolhidos ao final de cada dia pela pesquisadora.

\section{Procedimento de Análise dais Informações}

A organização e análise estatística dos dados foram processadas em computador através do programa MINITAB versão 12.2.

Para avaliar o grau de concordância dos escores, atribuídos pelas 4 enfermeiras, procedeu-se a análise quantitativa da variação do escore de cada uma das 4 enfermeiras (em si mesmo) para se verificar a existência de discrepância entre eles (Pearson).

\section{RESULTADOS E DISCUSSÃO}

Os dados apresentados a seguir referem-se ao teste de confiabilidade por equivalência entre diferentes avaliadores do instrumento de classificação de pacientes de Perroca ${ }^{(1)}$

Inicialmente, mostramos a caracterização dos pacientes classificados com este instrumento e a seguir, os escores atribuídos pelas enfermeiras a estes pacientes, segundo a complexidade assistencial.

Os 50 pacientes avaliados pelas enfermeiras durante este estudo eram predominantemente cio sexo masculino sendo $31(62 \%)$ e $19(38 \%)$ do sexo feminino. 
A faixa etária variou de 14 a 84 anos, com maior freqüência de pacientes nas faixas etárias de 31 a 40 anos e de 61 a 70 anos, como é possivel se observar pela tabela 1 .

Tabela 1- Distribuição dos pacientes classificados de acordo com faixa etária. São José do Rio Preto, 1999.

Na classificação de pacientes internados na UTI, os escores mínimos atribuídos pelas enfermeiras, de acordo com o quadro 1, variaram de 25 a 32 pontos, e os escores máximos foram 62 e 64.

\begin{tabular}{ccc}
\hline FAIXA ETIARIA & $\mathbf{N}^{\mathbf{0}}$ & $\mathbf{\%}$ \\
\hline $14-20$ & 2 & 4 \\
$21-30$ & 7 & 14 \\
$31-40$ & 14 & 28 \\
$41-50$ & 6 & 12 \\
$51-60$ & 3 & 6 \\
$61-70$ & 9 & 18 \\
$71-80$ & 5 & 10 \\
+ de 80 & 4 & .8 \\
\hline TOTAL & 50 & 100 \\
\hline
\end{tabular}

Quadro 1- Escores mínimos e máximos atribuídos pelas enfermeiras V, L, R e MS aos pacientes internados na UTI. São José do Rio Preto, 1999.

\begin{tabular}{ccccc}
\hline ESCORE & $\begin{array}{c}\text { ENF } \\
\text { V }\end{array}$ & ENF L & $\begin{array}{c}\text { ENF } \\
\mathbf{R}\end{array}$ & ENF MS \\
\hline MÍNIMO & 25 & 25 & 32 & 26 \\
\hline MÁXIMO & 64 & 64 & 64 & 62 \\
\hline
\end{tabular}

É interessante notar que o escore 25 corresponde a pacientes com tipo de Cuidados Mínimos e o escore 32 a pacientes com Cuidados Intermediários, os quais não deveriam fazer parte de uma Unidade de Cuidados Intensivos. Já os escores máximos obtidos nestas avaliações mostram uma variação mais estreita, de 62 a 64, identificando o perfil de complexidade assistencial de pacientes de Cuidados Intensivos, característicos de uma UTI.

De acordo com o quadro 2 evidencia-se, no que se refere à classificação dos 50 pacientes avaliados pelas enfermeiras, que $04(2 \%)$ deles foram categorizados como de Cuidados Mínimos; 33 (16,5\%) como sendo de Cuidados Intermediários; 79 (39,5\%) como Cuidados Semi-Intensivos e 84 (42\%) como de Cuidados Intensivos.

Quadro 2 - Distribuição do pacientes de UTI segundo categoria de cuidado, após aplicação do instrumento de classificação de pacientes de Perroca. São José do Rio Preto, 1999.

\begin{tabular}{|ccccccccccc|}
\hline CATEGORIA DE & \multicolumn{2}{c}{ ENF V } & \multicolumn{2}{c}{ ENF L } & \multicolumn{2}{c}{ ENF R } & \multicolumn{2}{c|}{ ENF MS } & TOTAL \\
\hline CUIDADO & $\mathbf{N}^{\circ}$ & $\mathbf{\%}$ & $\mathbf{N}^{\circ}$ & $\mathbf{\%}$ & $\mathbf{N}^{\circ}$ & $\%$ & $\mathbf{N}^{\circ}$ & $\%$ & $\mathbf{N}^{\circ}$ & $\%$ \\
\hline Mínimos & 01 & 2,0 & 02 & 4, & - & - & 01 & 2,0 & 04 & 2,0 \\
\hline Intermediários & 09 & 18,0 & 10 & 20,0 & 08 & 16,0 & 06 & $12,0$. & 33 & 16,5 \\
\hline Semi-intensivos & 19 & 38,0 & 17 & 34,0 & 22 & 44,0 & 21 & 42,0 & 79 & 39,5 \\
\hline Intensivos & 21 & 42,0 & 21 & 42,0 & 20 & 40,0 & 22 & 44,0 & 84 & 42,0 \\
\hline Total & 50 & 100,0 & 50 & 100,0 & 50 & 100,0 & 50 & 100,0 & 200 & 100,0 \\
\hline
\end{tabular}

A existência de pacientes de Cuidados Mínimos e Intermediários dentro de uma Unidade de Terapia Intensiva nos remete à reflexão se houve falha inicial quando da alocação destes pacientes nesta unidade, talvez por falta de utilização de um instrumento de avaliação mais criterioso, ou se os pacientes permaneceram nesta Unidade por problemas relacionados à deficiência de vagas nas Unidades de Internação.

Contudo, a presença de pacientes de Cuidados Semi-Intensivos mostra-se cabível, mesmo porque, nessa UTI, existem leitos destinados a tais pacientes.

\section{Estudo das Correlações (Pearson) entre os escores das enfermeiras e a discrepância}

A tabela 2 mostra o coeficiente de correlação obtido entre os escores totais atribuidos pelas enfermeiras avaliadoras e a discrepância existente entre eles. 
Tabela 2 - Coeficiente de Correlação de Pearson e respectivos valores p, entre os escores atribuídos pelas enfermeiras e a discrepância entre escores mínimos e máximos. São José do Rio Preto,1999.

$\begin{array}{cc}\text { ESCORES } & \text { ENFERMEIRA } \\ \text { TOTAL L } & 0,938 \\ \text { TOTAL R } & 0,945 \\ \text { TOTAL MS } & 0,917 \\ \text { DISCREPÂNCIA } & -0,227 \\ \text { Valor de p } & 0.112\end{array}$

Uma análise da variação dos escores totais de cada uma das 4 enfermeiras (tabela 2) revela que as correlações são altas e positivas entre os escores dados. No entanto, somente há correlação entre a discrepância de escore da enfermeira L, e esta correlação é negativa. A discrepância pode ser considerada como a diferença obtida entre o escore máximo e o escore mínimo.

Isto talvez possa ser explicado pelo fato da enfermeira L ter sido deslocada para a UTI, a fim de classificar os pacientes para este estudo. Estes resultados demonstram a importância do conhecimento das condições clínicas do paciente pela enfermeiraavaliadora na obtenção de dados legitimos e confiáveis.

É interessante notar que não há discrepância significativa na avaliação das demais enfermeiras que pertenciam à UTI durante o periodo da pesquisa. Em muitas das situações em que ocorre

\section{ENFERMEIRA R ENFERMEIRA MS}

$\begin{array}{ll}0,883 & \\ 0,872 & 0,958 \\ -0,396 & -0,194 \\ 0,004 & 0,177\end{array}$

$\begin{array}{lll}0,004 & 0,177 & 0,524\end{array}$

discrepância de resultados, os horários de avaliação são muito diferentes, tais como início e final do próprio turno (cerca de 6 horas de intervalo) e, em outras ocasiões mais de 6 horas de intervalo entre as mensurações. Isto contraria as orientações que havíamos fornecido às enfermeiras, ou seja, que as avaliações fossem realizadas nos horários mais próximos possiveis. Este intervalo acentuado nos horários de classificação pode ter captado mudanças no estado de saúde do paciente entre as classificações realizadas.

Outra condição que pode ter conduzido à discrepância seria a inabilidade da enfermeira em lidar com o instrumento, visto que não foi feita uma explicação detalhada do mesmo, pois a interpretação do instrumento faz parte do teste de confiabilidade.

Esses achados podem ser evidenciados no quadro 3. 
Quadro 3 - Apresentação dos horários de avaliação e a discrepância entre os escores dados pela enfermeiras. São José do Rio Preto, 1999

\begin{tabular}{|c|c|c|c|c|c|}
\hline $\mathbf{N}^{\circ}$ & HORA V & HORA L & HORA R & HORA MS & Disc \\
\hline 1 & 9: 30: 00, 00AM & $8: 45: 00,00 A M$ & 9:10:00,00AM. & $8: 55: 00$, OOAM & 10 \\
\hline 2 & $9: 15: 00,00 A M$ & 9:30:00,00AM & 9:05:00,00AM & $8: 30: 00,00 A M$ & 1 \\
\hline 3 & 8:30:00,00AM & 8:00:00,00AM. & 8:50:.00,00AM: & $9: 30: 00,00 A M$ & 9 \\
\hline 4 & 8:30:00,00AM & 8:30:00,00AM & 9:00:00,00AM & 9:15:00,00AM & 2 \\
\hline 5 & 10:30:00,00AM & $9: 30: 00,00 \mathrm{AM}$ & 9:05:00,00AM & 11:00:00,00AM & 6 \\
\hline 6 & $9: 15: 00,00 \mathrm{AM}$ & 9:00:00,00AM & $9: 15: 00,00 \mathrm{AM}$ & * & 3 \\
\hline 7 & $8: 50: 00,00 A M$ & $8: 30: 00,00 A M$ & $8: 55: 00,00 A M$ & $*$ & 1 \\
\hline 8 & $8: 30: 00,00 \mathrm{AM}$ & $8: 45: 00,00 A M$ & $8: 45: 00,00 A M$ & 10:40:00,00AM & 0 \\
\hline 9 & 12:40:00,00PM & $1: 30: 00,00 P M$ & 9:05:00.,00AM & 10:50:00,00AM & 9 \\
\hline 10 & 12:50:00,00PM & 12:50:00,00PM & 9:30:00,00AM & $11: 40: 00,00 A M$ & 1 \\
\hline 11 & 1:00:00,00PM & 1:00:00,00PM & $9: 15: 00,00 A M$ & 11:00:00,00AM & 5 \\
\hline 12 & $*$ & $12: 30: 00,00 \mathrm{PM}$ & 9:40:00,00AM & $11: 15$ 00, 00AM & 15 \\
\hline 13 & 1:05:00,00PM & $1: 05: 00,00 \mathrm{PM}$ & $9: 25: 00,00 A M$ & 11 30:00,00AM & 19 \\
\hline 14 & $12: 35: 00,00 P M$ & * & 11:50:00,00AM & $12: 35: 00,00 \mathrm{PM}$ & 0 \\
\hline 15 & $1: 00: 00,00 \mathrm{PM}$ & $12: 45: 00,00 \mathrm{PM}$ & 12:00:00,00PM & $12: 30: 00,00 \mathrm{PM}$ & 0 \\
\hline 16 & $1: 15: 00,00 \mathrm{PM}$ & * & $12: 10: 00,00 P M$ & * & 0 \\
\hline 17 & $1: 15: 00,00 P M$ & 1:10:00,00PM & $12: 20: 00,00 \mathrm{PM}$ & * & 0 \\
\hline 18 & 1:05:00,00PM & 1:20:00,00PM & 12:30:00,00PM & $*$ & 1 \\
\hline 19 & 12:50:00,00PM & $2: 40: 00,00 \mathrm{PM}$ & 12:40:00,00PM & $12: 45: 00,00 \mathrm{PM}$ & 4 \\
\hline 20 & $12: 40: 00,00 \mathrm{PM}$ & 6:30:00,00PM & 12:30:00,00PM & $12: 25: 00,00 P M$ & 3 \\
\hline 21 & $12: 20: 00,00 \mathrm{PM}$ & $6: 15: 00,00 \mathrm{PM}$ & $12: 20: 00,00 \mathrm{PM}$ & $12: 35: 00,00 \mathrm{PM}$ & 3 \\
\hline 22 & $1: 15: 00,00 \mathrm{PM}$ & 2:00:00,00PM & $12: 15: 00,00 \mathrm{PM}$ & 12:00:00,00PM & 3 \\
\hline 23 & $1: 00: 00,00 P M$ & 1:50:00,00PM & 12:00:00,00PM & $12: 10: 00,00 \mathrm{PM}$ & 0 \\
\hline 24 & $1: 20: 00,00 \mathrm{PM}$ & $2: 10: 00,00 P M$ & $12: 30: 00,00 \mathrm{PM}$ & $12: 20: 00,00 \mathrm{PM}$ & 2 \\
\hline 25 & $1: 30: 00,00 P M$ & $2: 30: 00,00 \mathrm{PM}$ & * & $12: 35: 00,00 \mathrm{PM}$ & 1 \\
\hline 26 & 12:50:00,00PM & $2: 45: 00,00 \mathrm{PM}$ & 12:50:00,00PM & $12: 45: 00,00 \mathrm{PM}$ & 0 \\
\hline 27 & 1:20:00,00PM & 5:00:00,00PM & 1:00:00,00PM & 7:05:00,00AM & 0 \\
\hline 28 & 2:00:00,00PM & 5:45:00,00PM & 12:40:00,00PM & 6:30:00,00AM & 0 \\
\hline 29 & $1: 30: 00,00 \mathrm{M}$ & 5:15:00,00PM & 12:20:00,00PM & $7: 15: 00,00 A M$ & 1 \\
\hline 30 & 1:40:00,00M & 6:00:00, 00РM & 12:50:00,00PM & $6: 55: 00,00 A M$ & 0 \\
\hline 31 & 1:00:00,00PM & 5:30:00,00PM & 12:30:00,00PM & $6: 40: 00,00 \mathrm{AM}$ & 0 \\
\hline 32 & 12:00:00,00PM & $1: 40: 00,00 \mathrm{PM}$ & 11:00:00,00AM & $11: 40: 00,00 \mathrm{AM}$ & 2 \\
\hline 33 & 12:00:00,00PM & $1: 30: 00,00 \mathrm{PM}$ & $11: 15: 00,00 A M$ & $11: 30: 00,00 \mathrm{AM}$ & 1 \\
\hline 34 & $12: 20: 00,00 \mathrm{PM}$ & $1: 15: 00,00 \mathrm{PM}$ & 11:20:00,00AM & $11: 15: 00,00 A M$ & 2 \\
\hline 35 & $12: 15: 00,00 \mathrm{PM}$ & 2:00:00,00PM & $11: 30: 00,00 A M$ & $11: 10: 00,00 A M$ & 2 \\
\hline 36 & $11: 40: 00,00 \mathrm{AM}$ & $1: 20: 00,00 \mathrm{PM}$ & $11: 10: 00,00 A M$ & $11: 20: 00,00 \mathrm{AM}$ & 2 \\
\hline 37 & 2:20:00,00PM & $6: 45: 00,00 \mathrm{PM}$ & 12:20:00,00PM & 12 55:00,00PM & 9 \\
\hline 38 & $2: 15: 00,00 \mathrm{PM}$ & 6:00:00,00PM & 12:45:00,00PM & * & 9 \\
\hline 39 & 12:10:00,00PM & 7:00:00,00PM. & 12:35:00, 00.PM & $12: 25: 00,00 P M$ & 23 \\
\hline 40 & $1: 10: 00,00 \mathrm{PM}$ & $7: 15: 00,00 P M$ & $12: 40: 00,00 P M$ & $12: 35: 00,00 \mathrm{PM}$ & 11 \\
\hline 41 & * & $6: 15: 00,00 P M$ & 12:50:00,00PM & & 7 \\
\hline 42 & * & 6:30:00,00PM & $12: 25: 00,00 \mathrm{PM}$ & $12: 45: 00,00 \mathrm{PM}$ & 7 \\
\hline 43 & 12:30: 00, $00 \mathrm{PM}$ & $1: 40: 00,00 \mathrm{PM}$ & $11: 10: 00,00 A M$ & 10:40:00,00AM & 0 \\
\hline 44 & 11:00:00,00AM & 1:50:00,00M & $11: 15: 00,00 A M$ & $10: 50: 00,00 A M$ & 0 \\
\hline 45 & 10:30:00,00AM & 1:15:00,00PM & 10:50:00,00AM & 11:00:00,00AM & 0 \\
\hline 46 & 10:15:00,00AM & 2:00:00,00AM & 9:00:00,00AM & 11:10:00, 00AM & 2 \\
\hline 47 & $11: 15: 00,00 A M$ & $2: 15: 00,00 P M$ & 9:05:00,00AM & $11: 20: 00,00 A M$ & 0 \\
\hline 48 & * & 2:30:00,00PM & 9:10:00,00AM & 11:30:00,00AM & 0 \\
\hline 49 & * & $2: 45: 00,00 \mathrm{PM}$ & $8: 50: 00,00 A M$ & 11:40:00, 00AM & 0 \\
\hline 50 & 12:00:00,00PM & 7:30:00,00PM & 11:00:00,00AM & 10:30:00,00AM & 1 \\
\hline
\end{tabular}


Kinley; Cronenwett(18) realizaram estudo em um hospital de ensino de grande porte, na Nova Inglaterra, utilizando uma amostra de 621 casos com o propósito de avaliar se havia necessidade de mensurações múltiplas, ou seja, em cada um dos três plantões diários, quando da utilização de SCP. Os resultados revelaram que uma classificação diária dos pacientes é considerada suficiente devido ao fato de não ser significante o número de pacientes que tiveram alterações no quadro durante os demais turnos do dia.

De acordo com estas autoras, estes dados podem ser explicados por não terem ocorrido modificações significativas nas condições dos pacientes nas 24 horas ou, ainda, porque as enfermeiras estariam copiando informações do plantão anterior. Contudo, não foram incluídas na amostra da pesquisa pacientes de cuidados intensivos.

Desta forma, os diferentes escores obtidos pelas enfermeiras quando classificando o mesmo paciente, podem ter ocorrido por causa de pequenas mudanças ocorridas nas necessidades de cuidado dos pacientes da UTI, quando houve longos intervalos de classificação, uma vez que estes valores encontram-se em zonas limitrofes, ou seja, os escores de uma enfermeira conduzem à classificação do paciente na categoria de cuidado, imediatamente superior ou inferior às demais.

Outro aspecto que emerge é a tendência, embora não significativa, entre os escores e a discrepância. Isto pode ser interpretado da seguinte forma : quanto menor for o escore dado por uma enfermeira, maior é a discrepância com alguma das outras e vice-versa.

\section{CONCLUSÃO}

Considerando-se os resultados obtidos nos testes de confiabilidade entre avaliadores a que foi submetido o instrumento de classificação de pacientes de Perroca $^{(1)}$ de uma maneira sucinta podemos destacar os seguintes aspectos :

- A variação dos escores totais de cada uma das 4 enfermeiras revelou que as correlações são altas e positivas entre os escores dados;

- Houve certa discrepância nos resultados que podem ser explicados pelo intervalo acentuado entre avaliações, captando mudanças no estado de saúde dos pacientes classificados ou por causa da inabilidade da enfermeira em lidar com o instrumento;

- Quanto menor for o escore dado por uma enfermeira, maior é a discrepância com alguma das outras e vice-versa;

Estes dados demonstram evidências suficientes que este instrumento de classificação de pacientes apresenta confiabilidade para ser utilizado na prática gerencial do enfermeiro como instrumento diagnóstico de categoria de cuidado a que o paciente pertence, bem como da carga de trabalho da equipe de enfermagem.

\section{REFERÊNCIAS BIBLIOGRÁFICAS}

(1)Perroca MG. Sistema de classificação de pacientes construção e validação de um instrumento. São Paulo, 1996. 93 p. Dissertação (Mestrado) - Escola de Enfermagem, Universidade de São Paulo.

(2)Galvão CM et al. A liderança do enfermeiro no século XXI: algumas considerações. Rev Esc Enferm USP, 1998; 32(4): 302-6.

(3)Alward RR. Patient classification systems: the ideal vs. reality. J Nurs Adm, 1983; 13:(2): 14-9.

(4)De Groot HA. Patient classification system evaluation. Part 1 :

Essential system elements. J Nurs Adm, 1989a; 19(6): 30-5.

(5) Van Slyck A. A systems approach to the management of nursing services - Part II: patient classification systems. Nurs Manag, 1991; 22(4): 23-5

(6)Perroca MG, Gaidzinski RR. Sistema de classificação de pacientes: construção e validação de um instrumento. Rev Esc Enferm USP, 1998; 32(2): 153-68.

(7)Horta WA. Processo de enfermagem. São Paulo: EPU, 1979.

(8)Giovannetti P Understanding patient classification systems. J Nurs Adm, 1979; 9(2): 4-9.

(9)Giovannetti P, Mayer GG. Building confidence in patient classification systems. Nurs Manag, 1984; 15(8): 31-4.

(10)Whitney JD, Killien MG. Establishing predictive validity of a patient classification system. Nurs Manag, 1987; 18(5): 80-6.

(11)Haas SA. Patient classification systems : a self-full filling prophecy. Nurs Manag, 1988; 19(5): 56-62.

(12)Williams MA. When you don't develop your own: validation methods for patient classification systems. Nurs Manag, 1988; 19(3): 91-6.

(13)De Groot HA. Patient classification system evaluation. Part 2: System selection implementation. J Nurs Adm, 1989b; 19(7): 24-30.

(14)Spinola AWP. Delfos: proposta tecnológica alternativa. São Paulo, Faculdade de Saúde Pública da USP, 1984.

(15)Polit DF, Hungler BP. Fundamentos de pesquisa em enfermagem. 3. ed. Porto Alegre, Artes Médicas, 1995a. cap.9, p. 199-219: Qualidade dos dados.

(16)Horn SD, Horn RA. Reliability and validity of the severity of illness index. Med Care, 1986; 24(2): 159-78.

(17)Polit DF, Hungler BP. Fundamentos de pesquisa em enfermagem. 3. ed. Porto Alegre, Artes Médicas, 1995b. cap.10, p. 223-67: Análise Quantitativa.

(18)Kinley J, Cronenwett LR. Multiple shift patient classification:

is it necessary? J. Nurs. Adm., v.17, n.2, p. 22-5, 1987

\section{Artigo recebido em 26/03/01}

Artigo aprovado em 24/07/02 